\title{
Structural Colors Enabled by Lattice Resonance on Silicon
}

\section{Nitride Metasurfaces}

Jhen-Hong Yang ${ }^{a}$, Viktoriia E. Babicheva ${ }^{b}$, Min-Wen Yuc , Tien-Chang Lu ${ }^{d}$, Tzy-Rong Line, and Kuo-Ping Chen ${ }^{f, t}$

anstitute of Photonic System, College of Photonics, National Chiao-Tung University, Tainan 71150, Taiwan

${ }^{b}$ Department of Electrical and Computer Engineering, University of New Mexico, Albuquerque, New Mexico 87131, United States

'Institute of Lighting and Energy Photonics, College of Photonics, National Chiao-Tung University, Tainan 71150, Taiwan

${ }^{\mathrm{d} D e p a r t m e n t}$ of Photonics, College of Electrical and Computer Engineering, National Chiao-Tung University, Hsinchu 30010, Taiwan

eDepartment of Mechanical and Mechatronic Engineering, National Taiwan Ocean University, Keelung 20224, Taiwan

fInstitute of Imaging and Biomedical Photonics, College of Photonics, National Chiao-Tung University, Tainan 71150, Taiwan

+ Corresponding Author: kpchen@nctu.edu.tw 


\section{- Refractive index of $\mathrm{Si}_{3} \mathrm{~N}_{4}$ and $\mathrm{TiO}_{2}$}

Figure $\mathrm{S} 1$ shows the refractive index of $\mathrm{Si}_{3} \mathrm{~N}_{4}$ and $\mathrm{TiO}_{2}{ }^{1,2}$ in our simulations. The refractive index of PECVD-Si $\mathrm{N}_{4}$ is fitted by the ellipsometer. Note that the scale of loss is $10^{-3}$.

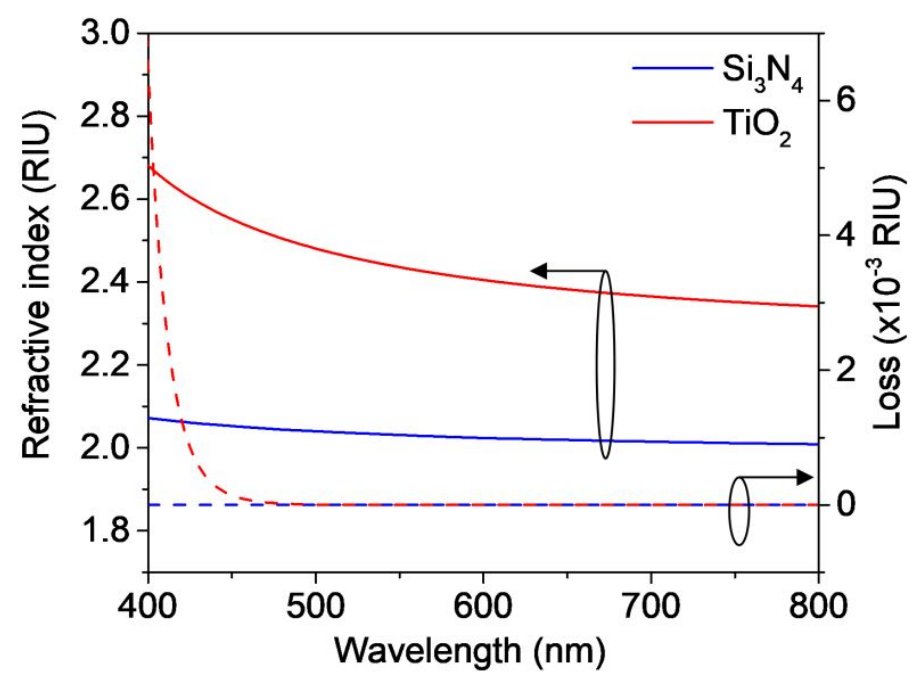

Figure S1. The refractive index of $\mathrm{Si}_{3} \mathrm{~N}_{4}$ and $\mathrm{TiO}_{2}$ used in the simulation.

\section{- Fabrication of $\mathrm{Si}_{3} \mathrm{~N}_{4}$ metasurfaces}

The silicon nitride thin film was deposited on a quartz substrate with a plasmaenhanced chemical vapor deposition (PECVD) system (Oxford Plasmalab System 100). Afterward, poly (methyl methacrylate) (PMMA)-A4 photoresist was spin-coated on the $\mathrm{Si}_{3} \mathrm{~N}_{4}$ thin film for further processing of the metasurface pattern. Conductive polymer (AR-PC 5090 by ALLRESIST) was spin-coated on the PMMA and then exposed by the E-beam lithography system (ELS-7500 EX by ELIONIX) to define the metasurface pattern. After photoresist developing, the chromium layer was deposited by a physical vapor deposition (PVD) system. Further, the PMMA was removed by acetone to form the chromium mask. The $\mathrm{Si}_{3} \mathrm{~N}_{4}$ layer was etched (EIS-700 by ELIONIX) at regions without chromium mask, and then the chromium hat was removed by chromium etchant (by Sigma-Aldrich). 


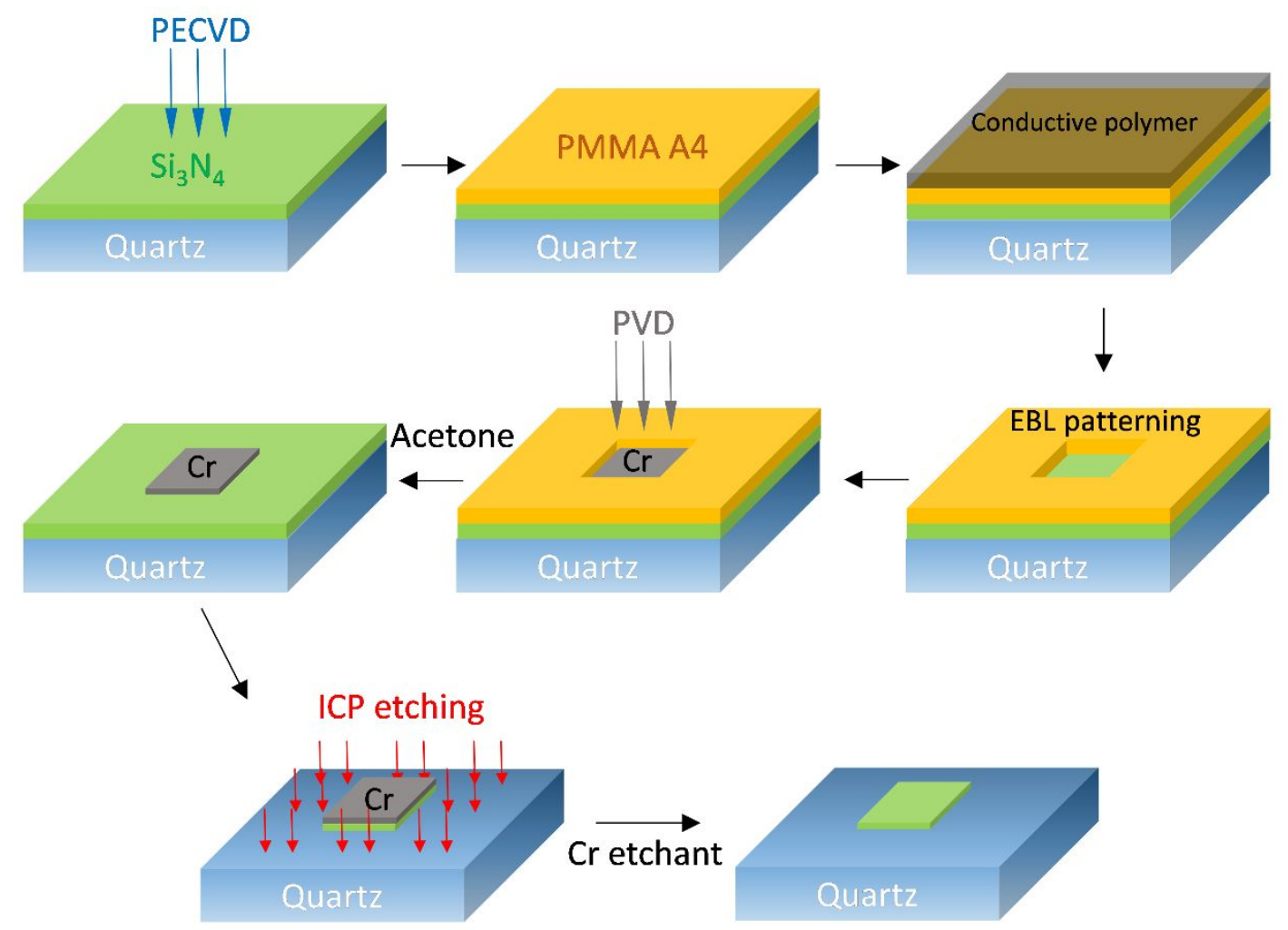

Figure S2. The fabrication flow chart of $\mathrm{Si}_{3} \mathrm{~N}_{4}$ metasurfaces.

\section{- Oblique reflection of $\mathrm{Si}_{3} \mathrm{~N}_{4}$ color pixels under $s$ - and $p$-polarization}

Since the viewing angle properties are important for display applications, the simulated oblique reflectance spectra of $\mathrm{Si}_{3} \mathrm{~N}_{4}$ color pixels for both $s$ - and $p$ polarization are represented in Figure $\mathbf{S 3}$, where the period, width, and height are 355 $\mathrm{nm}, 255 \mathrm{~nm}, 270 \mathrm{~nm}$, respectively. For s-polarization, the magnetic dipole lattice resonance (MDLR) is nearly independent at the incident angles (Figure S3a) smaller than 30 degrees, which is because of the effective period perpendicular to the magnetic field is fixed for MDLR. For $p$-polarization, MDLR is sensitively dependent on the incident angle (Figure S3b). The reason is that the effective period perpendicular to the magnetic field is increased by $p$-polarization oblique incidence, resulting in the red-shift of reflection peak from MDLR. Since the proposed design uses MDLR to create color pixels, the above discussion is in line with the principles and mechanisms of lattice resonance. To enlarge the viewing angle, the height of $\mathrm{Si}_{3} \mathrm{~N}_{4}$ nano-cuboid has to decrease down to $180 \mathrm{~nm}$ to prevent the Fano resonance of Fabery-Pérot resonance and MDLR. Figure S4 shows the oblique reflectance spectra in $s$ polarization are not affected even when the tilting angle is up to 60 degrees by changing the height of nano-cuboids to $180 \mathrm{~nm}$ (Figure S4). Overall, the proposed 
structural color pixels possess angle-independent property in s-polarization when the incident angle is smaller than 30 degrees. Furthermore, if the thickness of $\mathrm{Si}_{3} \mathrm{~N}_{4}$ nanocuboid is decreased to $180 \mathrm{~nm}$, the color pixels possess better angle-independent properties in s-polarization when the incident angle is smaller than 60 degrees, which implies utilizing the proposed metasurfaces with MDLR design is beneficial to displays applications. In addition, for $p$-polarization, the reflectance of samples with thickness

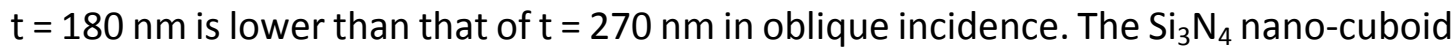
with $\mathrm{t}=180 \mathrm{~nm}$ cannot generate magnetic dipoles at wavelength $=550 \mathrm{~nm}$ when the electric field is not parallel to the top surface of nano-cuboid.

(a)

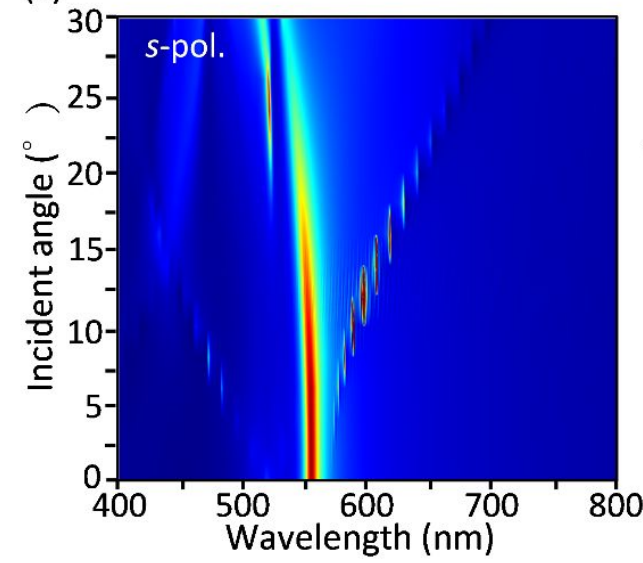

(b)

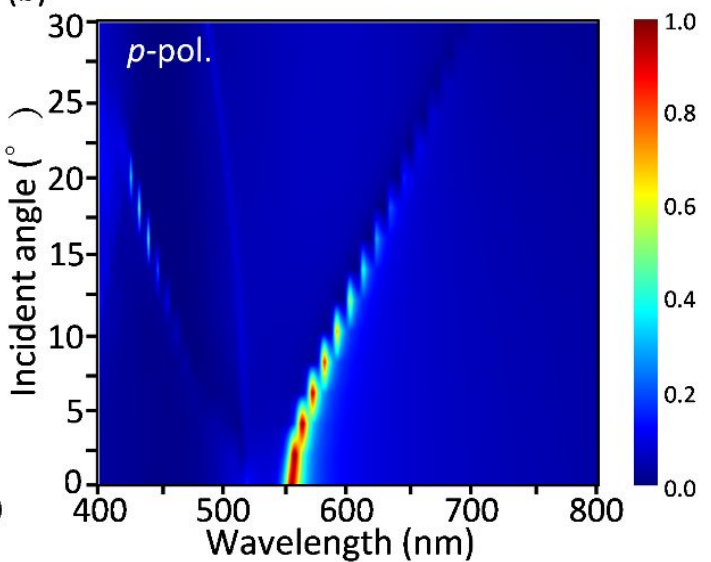

Figure S3. Simulated oblique reflectance spectra for $\mathrm{Si}_{3} \mathrm{~N}_{4}$ color pixels under (a) sand (b) $p$-polarizations for the thickness $\mathrm{t}=270 \mathrm{~nm}$.

(a)

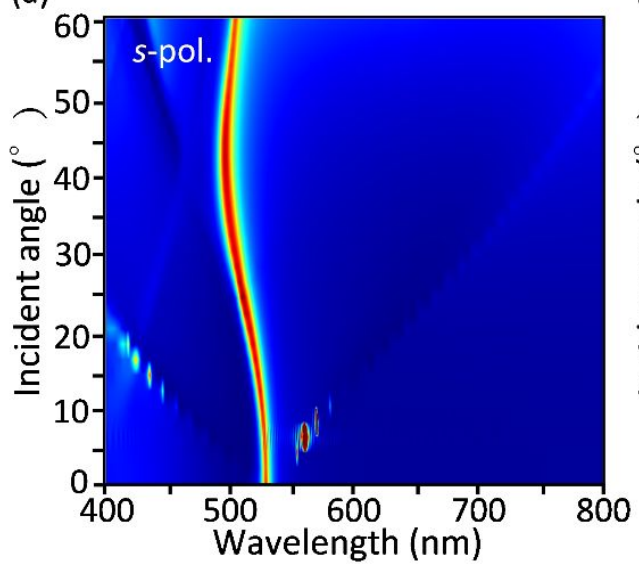

(b)

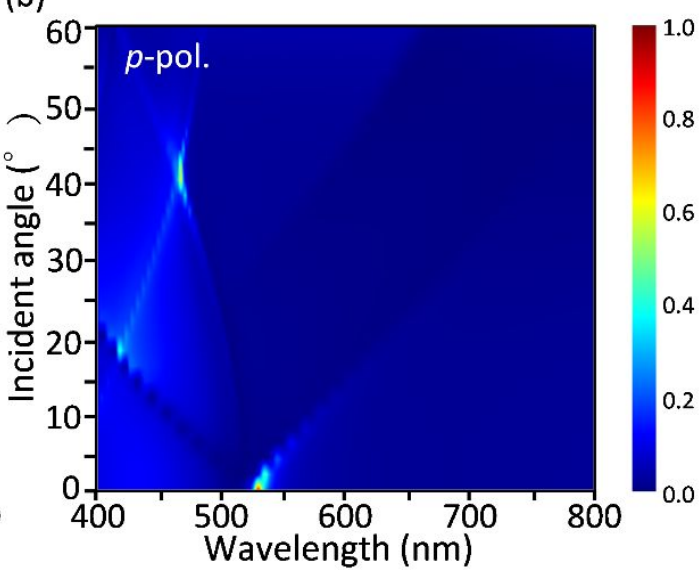

Figure S4. Simulated oblique reflectance spectra for $\mathrm{Si}_{3} \mathrm{~N}_{4}$ color pixels under (a) $s$ and (b) $p$-polarizations for the thickness $\mathrm{t}=180 \mathrm{~nm}$.

\section{- Images of $\mathrm{Si}_{3} \mathrm{~N}_{4}$ color pixels at different viewing angles}

Optical images of $\mathrm{Si}_{3} \mathrm{~N}_{4}$ color pixels with different oblique angles are experimentally 
analyzed. The objective lens is $x 5$ with numerical aperture 0.13 . In the proposed metasurfaces, the wavelengths of MDLR are designed at longer wavelengths than that of RA, so the optical diffraction phenomenon does not affect much in the color generation. The color pixels can also show the vivid colors when the oblique angle is at 30 degrees with a slight color difference.

(a)

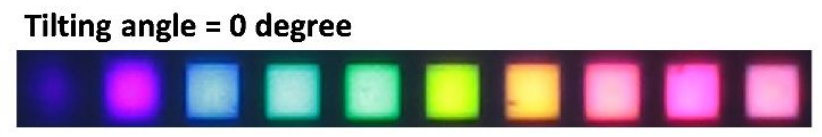

Tilting angle $\mathbf{=} \mathbf{3 0}$ degree

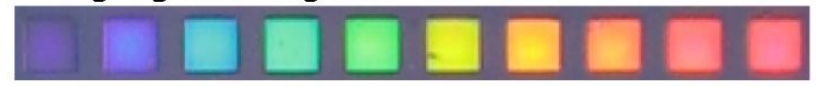

(b)
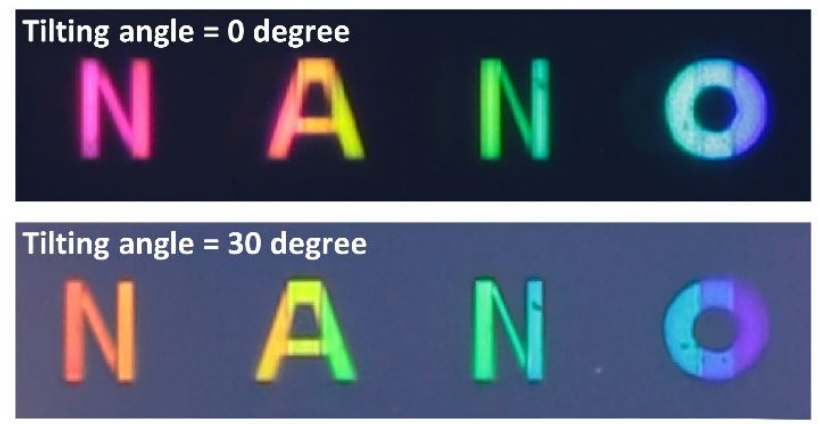

Figure S5. Optical images of $\mathrm{Si}_{3} \mathrm{~N}_{4}$ color pixels at different viewing angles.

\section{- The CIE map of $\mathrm{Si}_{3} \mathrm{~N}_{4}$ color pixels with lateral incidence}

The color of simulation and experiment spectra are plotted in the CIE 1931 chromaticity coordinates in Figure $\mathbf{S 6}$. The color spaces of $\mathrm{Si}_{3} \mathrm{~N}_{4}$ color pixels approach that of Adobe RGB.

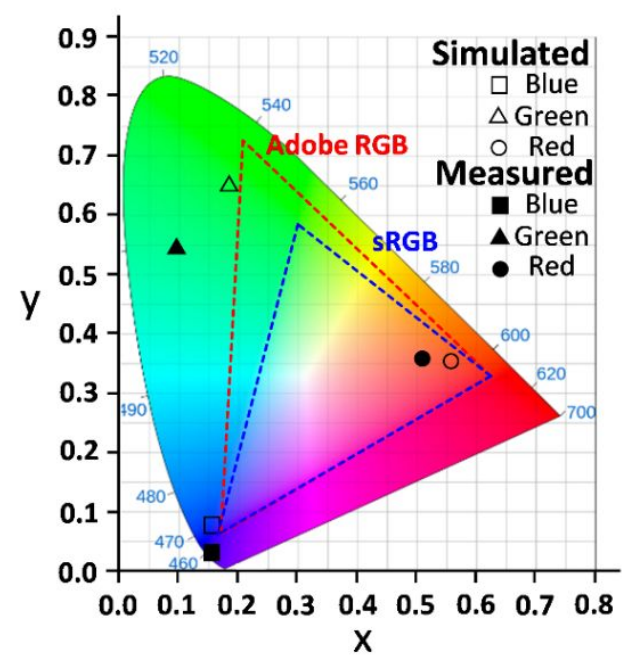


Figure S6. Color gamut of $\mathrm{Si}_{3} \mathrm{~N}_{4}$ metasurfaces with lateral incidence, $\mathrm{SRGB}$, and Adobe RGB color spaces in CIE 1931 chromaticity coordinates.

- The ripples in the scattering spectrum with the lateral incidence The ripples in scattering spectra are coming from the array number in the $y$-direction (see Figure S8). The simulated scattering spectra for $\mathrm{Si}_{3} \mathrm{~N}_{4}$ metasurfaces with 50 units in both $\mathrm{x}$ - and $\mathrm{y}$-directions are shown in Figure S7. The ripples beside the scattering peak are getting apparent when the number of units getting more and more.
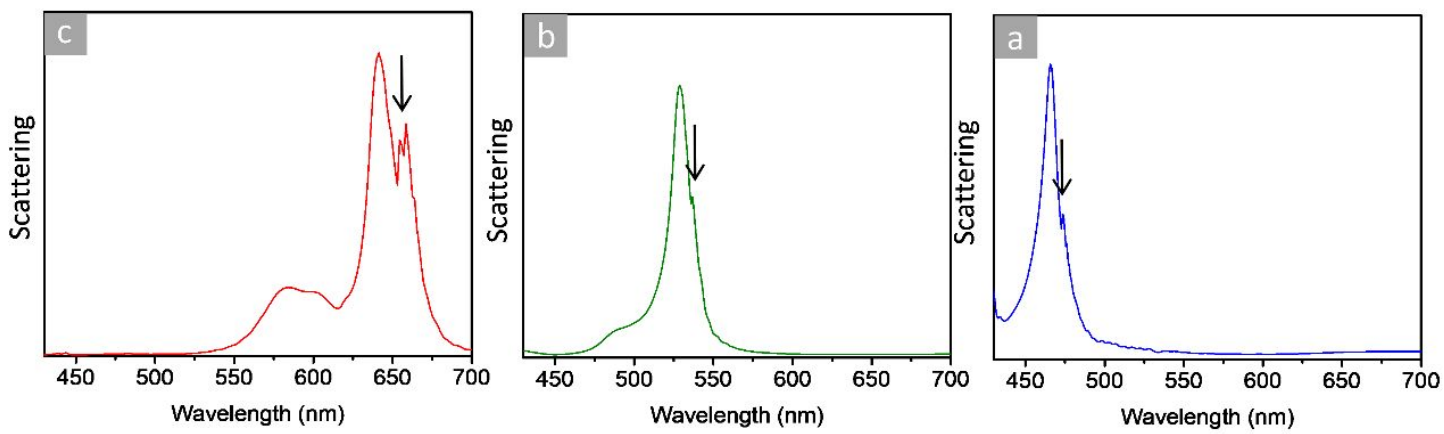

Figure S7. Normalized scattering spectra of (a) red, (b) green, and (c) blue. The case of $50 \times 50$ units in both $\mathrm{x}$ - and $\mathrm{y}$-directions.

\section{- Color pixel metasurfaces with TE- and TM-selectivity}

The schematics of the simulations of the lateral light incidence on color pixels are shown in Figure S8. The profile of the lateral incident light source is found as a solution for the waveguide and light propagation through the taper. The light comes out from the waveguide and illuminates the color pixel metasurface, which possesses 50 units of the nanoresonators in the $x$-direction and 16 units in the $y$-direction. The propagation mode of the waveguide can be separated in transverse electric (TE) and transverse magnetic (TM). Here, we define TE (TM) polarization as the light incidence where the electric field is along the $y(z)$-direction. The TE and TM selectivity are shown in Figure S9. The polarization ration scattering with TM incidence and its high values in some spectral regions are shown in Figure S10. 


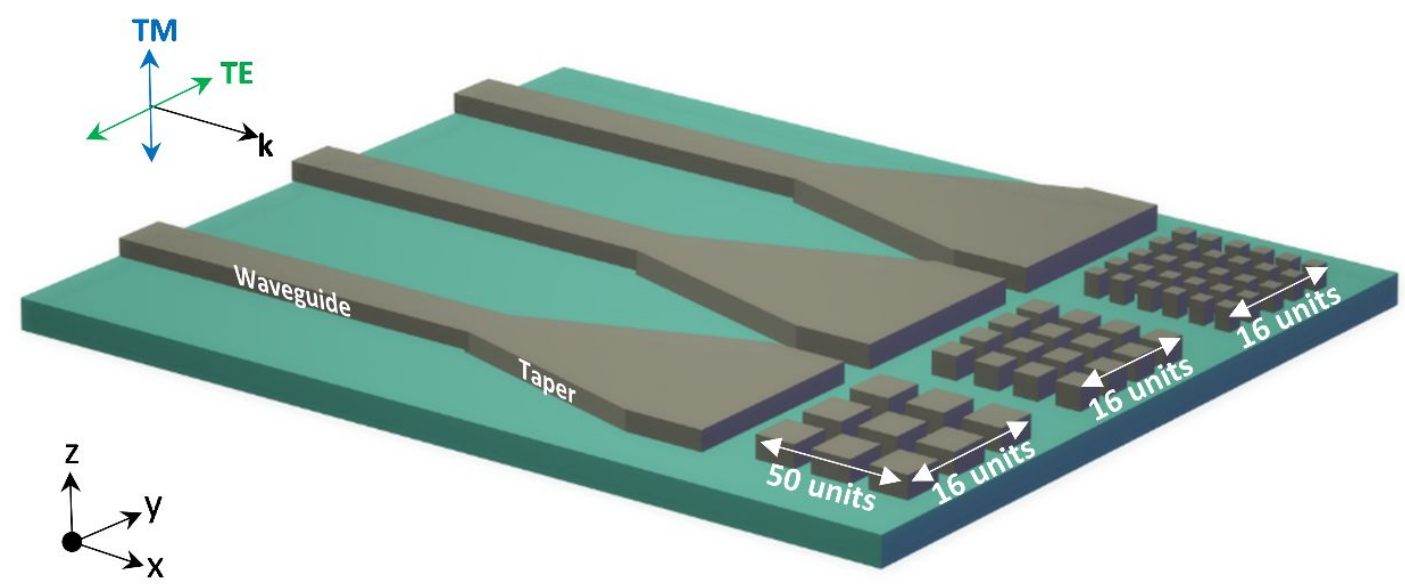

Figure S8. The schematics of waveguides with tapers and color metasurfaces in the FDTD simulations.
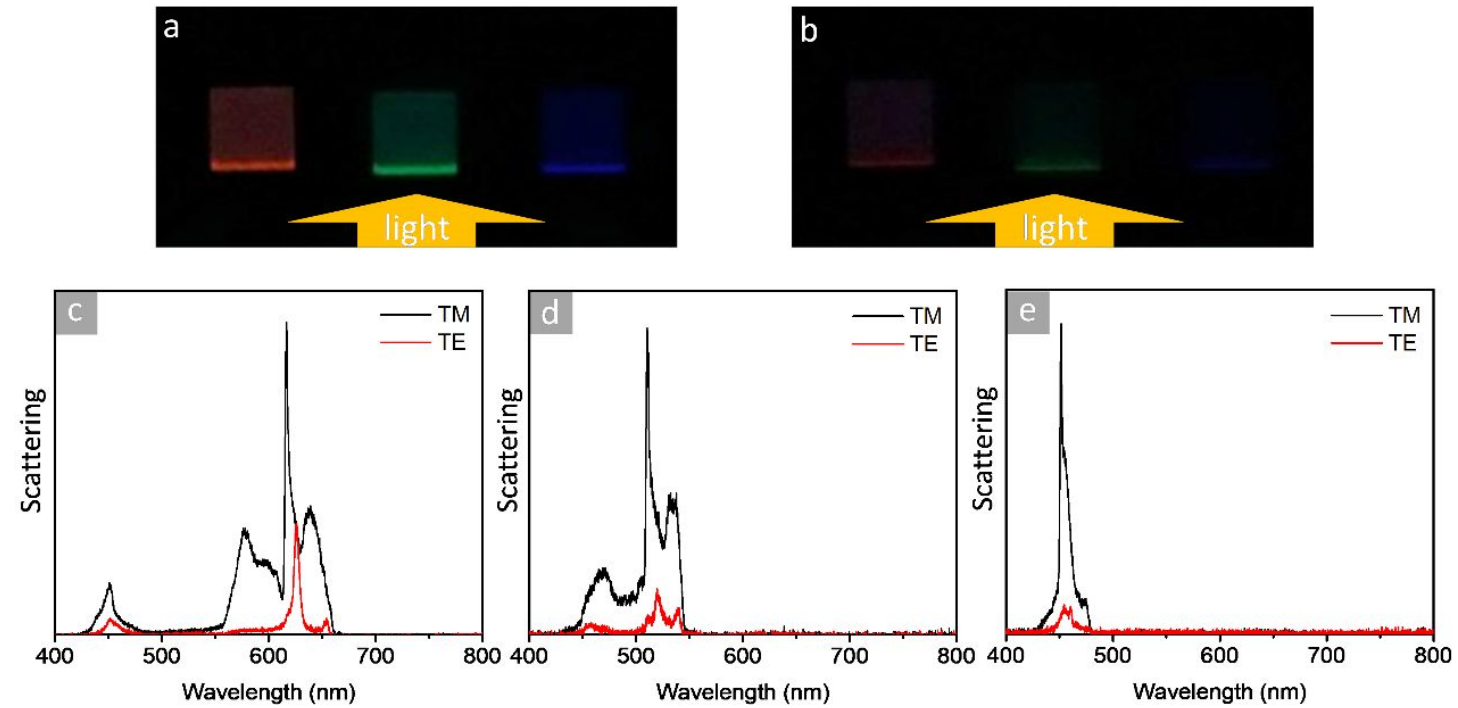

Figure S9. Optical images of color pixels with the lateral incidence of (a) TM and (b) TE. Normalized scattering spectra of (c) red, (d) green, and (e) blue with TM and TE polarizations in arbitrary units. 

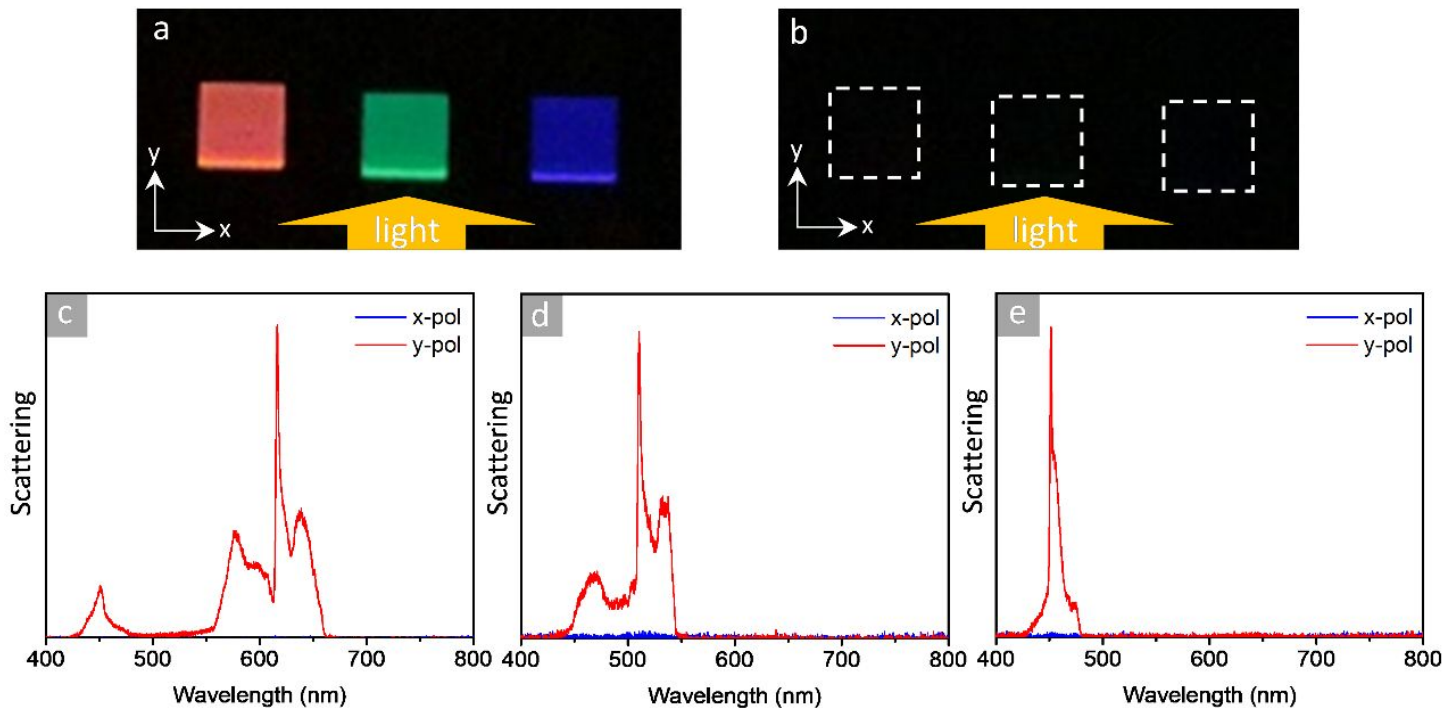

Figure S10. Optical images for TM incidence and analysis in (a) $y$ - and (b) $x$ polarizations. Normalized scattering spectra of (c) red, (d) green, and (e) blue in x- and $\mathrm{y}$-polarizations of light in arbitrary units.

\section{- References}

1. Ratzsch, S.; Kley, E.-B.; Tünnermann, A.; Szeghalmi, A., Influence of the Oxygen Plasma Parameters on the Atomic Layer Deposition of Titanium Dioxide. Nanotechnology 2014, 26, 024003.

2. Siefke, T.; Kroker, S.; Pfeiffer, K.; Puffky, O.; Dietrich, K.; Franta, D.; Ohlídal, I.; Szeghalmi, A.; Kley, E. B.; Tünnermann, A., Materials Pushing the Application Limits of Wire Grid Polarizers Further into the Deep Ultraviolet Spectral Range. Adv. Opt. Mater. 2016, 4, 1780-1786. 